\title{
$\mathrm{J}$

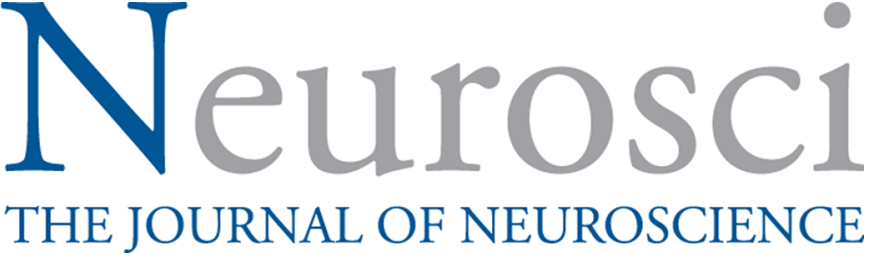

February 26, $2020 \bullet$ Volume 40 Number $9 \bullet$ www.jneurosci.org

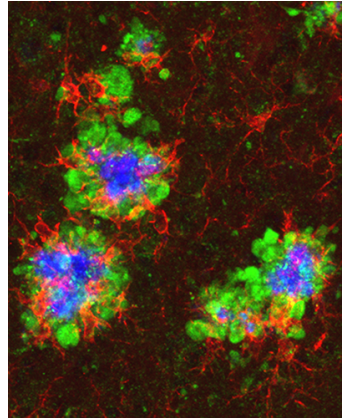

Cover legend: This image shows amyloid plaques (blue, methoxy-X04) surrounded by dystrophic, swollen axons that accumulate lysosome precursors (green, anti-Lamp1) in cortex of a mouse model of Alzheimer's disease (PS2APP mouse). Microglia (red, anti-Iba1) also cluster around the plaques and limit axonal damage. Loss of TREM2, a gene linked to Alzheimer's disease risk and expressed by microglia, elevates the level of soluble $A \beta$ in its toxic forms and exacerbates the axonal damage. For more information, see the article by Meilandt et al. (pages 1956-1974).

\section{This Week in The Journal}

\section{Journal Club}

1790 Two Routes to Incidental Memory under Arousal: Dopamine and Norepinephrine John Thorp, David Clewett, and Monika Riegel

1793 Heterosynaptic Plasticity in Cortical Interneurons Léa Caya-Bissonnette

\section{Research Articles}

\section{CELLULAR/MOLECULAR}

1795 The Origin of Physiological Local mGluR1 Supralinear $\mathrm{Ca}^{2+}$ Signals in Cerebellar Purkinje Neurons

Karima Ait Ouares and Marco Canepari

\section{DEVELOPMENT/PLASTICITY/REPAIR}

1810 Longitudinal Development of Brain Iron Is Linked to Cognition in Youth Bart Larsen, Josiane Bourque, Tyler M. Moore, Azeez Adebimpe, Monica E. Calkins, Mark A. Elliott, Ruben C. Gur, Raquel E. Gur, Paul J. Moberg, David R. Roalf, Kosha Ruparel, Bruce I. Turetsky, Simon N. Vandekar, Daniel H. Wolf, Russell T. Shinohara, and Theodore D. Satterthwaite

1819 Conserved Tao Kinase Activity Regulates Dendritic Arborization, Cytoskeletal Dynamics, and Sensory Function in Drosophila Chun $\mathrm{Hu}$, Alexandros K. Kanellopoulos, Melanie Richter, Meike Petersen, Anja Konietzny, Federico M. Tenedini, Nina Hoyer, Lin Cheng, Carole L.C. Poon, Kieran F. Harvey, Sabine Windhorst, Jay Z. Parrish, Marina Mikhaylova, Claudia Bagni, Froylan Calderon de Anda, and Peter Soba

\section{SYSTEMS/CIRCUITS}

1834 Spatial Arrangement Drastically Changes the Neural Representation of Multiple Visual Stimuli That Compete in More Than One Feature Domain Steven Wiesner, Ian W. Baumgart, and Xin Huang

1849 Repeated Exposure to Multiple Concurrent Stresses Induce Circuit Specific Loss of Inputs to the Posterior Parietal Cortex

Yaaqov Libovner, Mona Fariborzi, Daim Tabba, Ali Ozgur, Tamara Jafar, and Gyorgy Lur 
1862 Unique Spatial Integration in Mouse Primary Visual Cortex and Higher Visual Areas Kevin A. Murgas, Ashley M. Wilson, Valerie Michael, and Lindsey L. Glickfeld

1874 Neural Mechanisms Underlying High-Frequency Vestibulocollic Reflexes In Humans And Monkeys

Patrick A. Forbes, Annie Kwan, Brandon G. Rasman, Diana E. Mitchell, Kathleen E. Cullen, and Jean-Sébastien Blouin

1888 Phase-Specific Motor Efference during a Rhythmic Motor Pattern Ignacio Alonso, Agustín Sanchez Merlinsky, and Lidia Szczupak

\section{BEHAVIORAL/COGNITIVE}

1897 Vaporized Cannabis Extracts Have Reinforcing Properties and Support Conditioned Drug-Seeking Behavior in Rats

Timothy G. Freels, Lydia N. Baxter-Potter, Janelle M. Lugo, Nicholas C. Glodosky, Hayden R. Wright, Samantha L. Baglot, Gavin N. Petrie, Zhihao Yu, Brian H. Clowers, Carrie Cuttler, Rita A. Fuchs, Matthew N. Hill, and Ryan J. McLaughlin

1909 Sleep Spindles Promote the Restructuring of Memory Representations in Ventromedial Prefrontal Cortex through Enhanced Hippocampal-Cortical Functional Connectivity

Emily Cowan, Anli Liu, Simon Henin, Sanjeev Kothare, Orrin Devinsky, and Lila Davachi

1920 Cortical Overlap and Cortical-Hippocampal Interactions Predict Subsequent True and False Memory

Erik A. Wing, Benjamin R. Geib, Wei-Chun Wang, Zachary Monge,

Simon W. Davis, and Roberto Cabeza

\section{NEUROBIOLOGY OF DISEASE}

1931 Longitudinal Basal Forebrain Degeneration Interacts with TREM2/C3 Biomarkers of Inflammation in Presymptomatic Alzheimer's Disease

Taylor W. Schmitz, Hermona Soreq, Judes Poirier, and R. Nathan Spreng

1943 Elevated TRPV4 Levels Contribute to Endothelial Damage and Scarring in Experimental Spinal Cord Injury

Hemant Kumar, Chang Su Lim, Hyemin Choi, Hari Prasad Joshi, Kyoung-Tae Kim, Yong Ho Kim, Chul-Kyu Park, Hwan Myung Kim, and In-Bo Han

1956 Trem2 Deletion Reduces Late-Stage Amyloid Plaque Accumulation, Elevates the A $\beta 42: A \beta 40$ Ratio, and Exacerbates Axonal Dystrophy and Dendritic Spine Loss in the PS2APP Alzheimer's Mouse Model

William J. Meilandt, Hai Ngu, Alvin Gogineni, Guita Lalehzadeh, Seung-Hye Lee, Karpagam Srinivasan, Jose Imperio, Tiffany Wu, Martin Weber, Agatha J. Kruse, Kimberly L. Stark, Pamela Chan, Mandy Kwong, Zora Modrusan, Brad A. Friedman, Justin Elstrott, Oded Foreman, Amy Easton, Morgan Sheng, and David V. Hansen

1975 Mitochondrial Dysfunction Combined with High Calcium Load Leads to Impaired Antioxidant Defense Underlying the Selective Loss of Nigral Dopaminergic Neurons

Konrad M. Ricke, Thomas Paß, Sammy Kimoloi, Kai Fährmann, Christian Jüngst, Astrid Schauss, Olivier R. Baris, Marijana Aradjanski, Aleksandra Trifunovic, Therese M. Eriksson Faelker, Matteo Bergami, and Rudolf J. Wiesner 
Erratum: The article "Moment-to-Moment BOLD Signal Variability Reflects Regional Changes in Neural Flexibility across the Lifespan," by Jason S. Nomi, Taylor S. Bolt, C.E. Chiemeka Ezie, Lucina Q. Uddin, and Aaron S. Heller, appeared on pages 5539-5548 of the May 31, 2017 issue. An erratum for this article appears on pp. 1975-1980.

Persons interested in becoming members of the Society for Neuroscience should contact the Membership Department at membership@sfn.org or 202-962-4911.

For current submission policies and manuscript preparation guidelines, authors should refer to our Information for Authors at https://www.jneurosci.org/content/information-authors.

Manuscripts should be submitted online at https://jneurosci.msubmit.net. Please contact the Central Office with any questions at jn@sfn.org or 202-962-4000. 